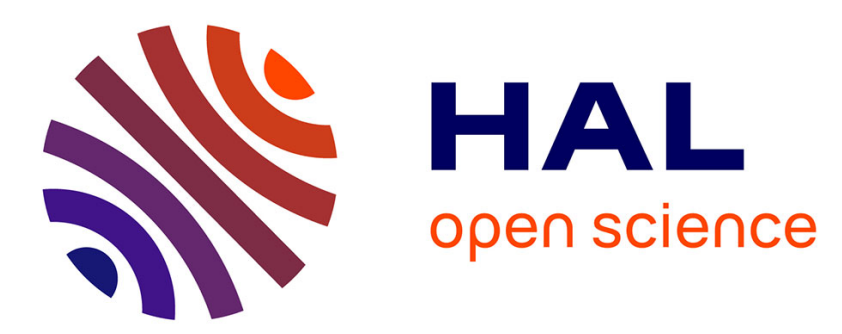

\title{
Steering and Lateral Motorcycle Dynamics Estimation: Validation of Luenberger LPV Observer Approach
}

Pierre-Marie Damon, Dalil Ichalal, Hichem Arioui

\section{To cite this version:}

Pierre-Marie Damon, Dalil Ichalal, Hichem Arioui. Steering and Lateral Motorcycle Dynamics Estimation: Validation of Luenberger LPV Observer Approach. IEEE Transactions on Intelligent Vehicles, 2019, 4 (2), pp.277-286. 10.1109/TIV.2019.2904384 . hal-02415924

\section{HAL Id: hal-02415924 \\ https://hal.science/hal-02415924}

Submitted on 19 Dec 2019

HAL is a multi-disciplinary open access archive for the deposit and dissemination of scientific research documents, whether they are published or not. The documents may come from teaching and research institutions in France or abroad, or from public or private research centers.
L'archive ouverte pluridisciplinaire HAL, est destinée au dépôt et à la diffusion de documents scientifiques de niveau recherche, publiés ou non, émanant des établissements d'enseignement et de recherche français ou étrangers, des laboratoires publics ou privés. 


\title{
Steering and Lateral Motorcycle Dynamics Estimation: Validation of Luenberger-like LPV Observer Approach
}

\author{
Pierre-Marie Damon, Dalil Ichalal and Hichem Arioui
}

\begin{abstract}
In this paper a validation of the Luenberger-like nonlinear observer, proposed in one of our recent work, is performed by means of a commercial motorcycle simulator. The previous paper has introduced the design of a nonlinear Luenberger observer to simultaneously estimate both the motorcycle lateral dynamics and the rider action. The vehicle lateral motion is described with a two-body linear model which takes into account the longitudinal speed variation as a time-varying parameter. Then, the Takagi-Sugeno approach combined to the Lyapunov theory, linear matrix inequalities tools and $L_{2}$-gain property are used to demonstrate the observer's convergence through an input-to-error stability. Finally, the effectiveness and the robustness of the observer are tested on three different realistic riding scenarios by means of the well-know multibody simulator BikeSim. Finally, the estimation performances are compared to previous works.
\end{abstract}

Index Terms-Motorcycle, Observation, Luenberger, Validation and Simulator.

\section{INTRODUCTION}

$\mathbf{N}$ OWADAYS Powered Two-Wheeled Vehicles (P2WV) play an important role in our society. Their compact size, their abilities to avoid congestion and to be easily parked, their low fuel consumption and their attractive selling price explain why motorcycles are one of the most popular mean of transportation. Although $\mathrm{P} 2 \mathrm{WV}$ are common on all continents, their use is much higher in Asian countries than elsewhere. In 2015, in Thailand, Vietnam, Indonesia and Malaysia almost $90 \%$ of the households had a motorcycle or a scooter which represented around $70 \%$ of the total number of registered vehicles in each country [1].

Unfortunatly, the growing number of P2WT users, inevitably, leads to an increasing amount of motorcycle accidents and related fatal injuries which alarms some organizations. According to the World Health Organization (WHO) in Global status report on road safety 2015 [2]: "Motorcyclist safety must be prioritized too". For several years rider accidents are became a major social concern because they formed one of the most vulnerable group of road users. Nearly a quarter of all road traffic deaths are among motorcyclists in the world. Despite of the human cost of road accidents there are real economic stakes hidden behind these issues. In France, during

All the authors are with IBISC Laboratory, University of Paris-Saclay, France. Mail: \{pierre-marie.damon, dalil.ichalal, hichem.arioui\}@univ-evry.fr

This work is supported by National Agency of Research under the framework VIROLO++.

Manuscript received April 19, 2005; revised August 26, 2015. the year 2015, the lack of road safety has cost more than 40 billion $€$ which represented $2.2 \%$ of the GDP [3].

The P2WV accidents are such important subject in our societies that specific organizations work on the understanding of the P2WV accidents as Motorcycle Accidents In Depth Study (MAIDS). MAIDS, which is an European non-profit organization, has investigated in detail no less than 921 motorcycle accidents in five European countries during one year [4]. This study highlights clear results:

- The main primary contributing factors in the accidents involving motorcycle were the riders $(37.4 \%)$ and the other vehicle (OV) drivers $(50.5 \%)$

- Main rider contributions to accident causality are presented in table I.

- In $32 \%$ there is a loss of control of the P2WV, it was mostly related to braking and a subsequent change in vehicle dynamics.

- Infrastructure, weather, mechanical problems are also identified factors responsible of accidents.

\begin{tabular}{|c|c|}
\hline Human factor & Contribution \\
\hline Traffic strategy & $32.2 \%$ \\
\hline Traffic-scan error & $27.7 \%$ \\
\hline Visual obstructions & $18.5 \%$ \\
\hline Speed difference & $18 \%$ \\
\hline Rider inattention & $10.6 \%$ \\
\hline
\end{tabular}

Table I: Rider contributions to accident causation

This paper is organized as follows: section 2 presents the motivations and states the problem. Then, section 3 reminds the basis of motorcycle modeling whereas section 4 deals with the main steps of the observer design. In section 5 , results of validation have been discussed. Finally, section 6 ends this paper through a conclusion.

\section{Motivation AND CONTEXT}

In the past, the first action established by many governments to answer to the concern of road fatalities was to push up the use of passive safety systems such as the seat belt in our Powered Four-Wheeled Vehicle (P4WV) or the helmet for the $\mathrm{P} 2 \mathrm{WV}$ riders (researches [2] have shown that wear a helmet can reduce the risk of death by almost $40 \%$ and the risk of severe injury by approximately 70\%). Depth investigations as MAIDS have highlighted that human is one of most contributing factors to accident causality especially riders who are responsible up to $37.4 \%$ of the total number of accidents 
involving at least one P2WV. Moreover, a loss of control of the motorcycle happens in $32 \%$ among all the P2WV accidents. This finding is very crucial because adequate systems could help the rider to avoid a loss of control and hence the accidents. That is why there is a real challenge to develop Advanced Rider Assistance Systems (ARAS) to assist the rider during dangerous riding scenarios and to avoid critical situations. It is very encouraging because similar systems equip our car for almost 10 years and they have significantly contributed to decrease the number of road accidents.

Lot of specificities of P2WV do not allow the motorcycle makers to use the already existing systems for P4WV and it explains the delay in the development of ARAS. The complexity of P2WV motions especially the lateral dynamics which involves yaw, roll, steering angles and lateral motion, the extreme load transfer phenomena (wheelie and stoppie), the ratio between the mass of the rider and the vehicle, their compact size, their cheaper cost, are undeniable obstacles to the development of such systems. Nevertheless, for the last 3 years, several ARAS have been proposed on the P2WV market. One can cite: the Antilock Braking System (ABS), the Electronic Stability Control (ESC), the Traction Control System (TCS) or the Motorcycle Stability Control (MSC). Unfortunately, most of them are installed only on high-end motorcycles.

In general, active and semi-active safety systems for vehicle work in a similar way, they are based on a mathematical model of the vehicle. Their efficiency is often closely linked to the capability of the model to be faithful regarding the real dynamics. The rich literature dealing with motorcycle modeling demonstrates how much the topic has been addressed for almost 50 years. However, very few works discuss the complete dynamics model of P2WV, they are often separated into 2 categories: "In-plane" describing the longitudinal dynamics and "Out-of-plane" for the lateral dynamics. It exists a large diversity of P2WV model whose the most simple is the single body one similar to an inverted pendulum. However, much more complex models have been developed like in [5] or in [6] where the motorcycle is divided into 8 different bodies (the fork, the steering mechanism, the front and rear wheels, the swing arm, etc). Although these multibody models are very accurate and reliable regarding the real P2WV dynamics, it is quite impossible to use them to design any algorithm for ARAS because of their intrinsic complexity with strong nonlinearities. Moreover, describe the vehicle with several bodies involves to determine all the parameters relative to each body especially the static and the dynamic features. It is sometimes very difficult to measure some of these parameters like the inertia. In this case, identification approaches as in [7] can be used to determine the corresponding parameters. Hence, in addition to the intrinsic complexity, the difficulty to identify all the needed parameters make them not adapted for ARAS design but very useful tools to proceed in a first validation of ARAS before testing them on real vehicles. Note that the commercial motorcycle simulator BikeSim (BS) which is used below to perform the validation is based on a 8 bodies mathematical model. Fortunately, intermediate models have been developped like in [8], [9] or the well-know model in
[10]. In our work and more generally in the literature, the works dealing with algorithm design for motorcycle dynamics state estimation and control purposes use the Sharp' 71 model. It is popular because with its two-body description it is a great compromise between simplicity and ability to catch all the lateral dynamics phenomena. Moreover, considering only two bodies considerably reduces the number of parameters to be identified.

Obviously, in addition to the choice of the mathematical model, the success of ARAS or ADAS is highly dependent of the embedded electronics systems. Most of them are based on the same pattern, they use an Electronic Control Unit (ECU), a whole of sensors and according to the kind of system some actuators. That is why ARAS currently equipped only premium vehicles because of their costs, their reliability, their integration constraints, etc. This paper aims to bypass these problems by introducing an observation algorithm to estimate the lateral dynamics. It allows to replace some sensors, doesn't need any mechanical maintenance, is directly implemented into the ECU, is free of integration constraint and is cheaper. Moreover, the proposed observer enables to estimate unmeasurable states like the tire forces or the rider action. Indeed, the rider torque applied on the handlebar is a typical unmeasurable information because even if strain gauges can give an idea about it, it is impossible to separate the rider contribution from the road aligning action contribution. While self-driving P4WV vehicles appears on the roads, autonomous P2WV are still in development [11],[12], [13]. In this kind of application observers are very useful because it is crucial to understand the steering torque dynamics since the rider action is replaced with an electric motor. The observers for the P2WV dynamics estimation have been seriously studied for 10 years. In 2008, precursor work was published about state estimation for motorcycles in [14]. In a first time, researchers have commonly addressed the estimation of the lean angle [15], [16], [17] and rapidly the aim was to estimate the whole dynamics states [18], [19] and more recently in [20] and [21]. These works dealing with P2WV dynamics estimation highlight the strong potential of these techniques. In [22] or in [23] authors have already proposed a validation of their observation works by means of BS whereas in [24] and [17] experimental tests have been performed but only roll angle estimation was considered. In [15] authors have proposed a more complete experimental investigation to validate estimated states with extended Kalman filter. Nevertheless, regarding the number of works addressing motorcycle dynamics observation few are validated with experimental tests or with a high performance simulator like BS.

The main contribution of this paper is the validation of the Luenberger-like nonlinear observer proposed in [20] with the P2WV commercial simulator BikeSim (BS). In [20], the authors have only discussed the performances of the approach by testing the algorithm on the simple linear twobody model used for the observer design. These first results have shown the potential of the proposed observer and this paper aims to confirm it with realistic simulations. Moreover, the performances comparison based on the Root Mean Square Error (RMSE) is performed with previous works dealing with 
P2WV estimation especially [25] and [26]. In addition, to be as close as possible to real experimentation, real sensors have been considered during the observer's design by introducing measurement noises in the observation equation. Let us remind that with its very simple design, its ability to rapidly and simultaneously estimate the lateral dynamics and the rider action without any restriction on the longitudinal speed, this observer turns out to be a perfect candidate for real time applications.

\section{MOTORCYCLE DYNAMICS}

This section aims to remind the main steps of the development of the P2WV mathematical model used for observer synthesis. Let us recall that the dynamics equations are derived in the reference frame whose the origin is the projection of the gravity center on the road and its Z-axis is perpendicular to the road. Notice that this reference frame is different from the body-fixed frame which is attached to the P2WV. Note that, the notation $X_{b f}$ is used when the dynamics state $X$ is expressed in the body-fixed frame. For those expressed in the reference frame there is no specific notation.

\section{A. Linearized two-body Model}

Let us remind that the model discussed below is based on the well-know Sharp' 71 model initially presented in [10]. In his work, the author has modeled the motorcycle as a set of two rigid bodies joined by the steering mechanism. The front body includes the front wheel, the fork and the steering mechanism whereas the rear body involves all the remaining parts of the P2WV (main frame, rear wheel, swing arm, etc) and the rider which is considered rigidly fixed to the rear frame. Figure 1 illustrates the geometric features of the two-body model. Note that the points $G_{f}$ and $G_{r}$ denote respectively the location of the front and rear gravity centers. For more details, please refer to $[10]$.

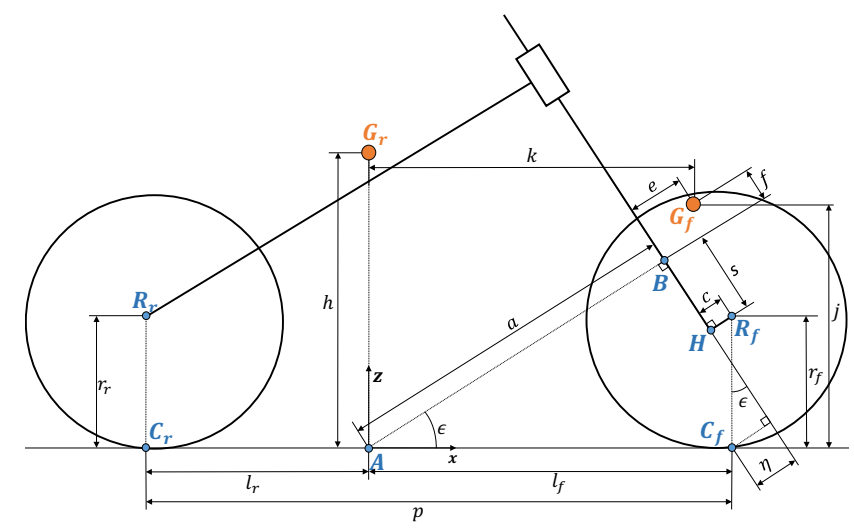

Figure 1: Sharp' two-body motorcycle kinematics

This model provides a total of 4 Degrees Of Freedom (DOF): the roll $\phi$, the yaw $\psi$, the steering $\delta$ and the lateral motion $v_{y}$. The pitch dynamics is neglected into this model.
Note that this assumption is important to derive the relationship between the angular rates in the body-fixed frame and the reference frame. Although this model allows to catch the main part of the lateral dynamics, it does not take into account any tire contribution. In [10], the author has also demonstrated how much it is essential to consider the tire forces since their dynamics play a crucial role into the vehicle stability. To this end, the tire relaxation phenomenon is commonly considered. It is defined by the following expression:

$$
\frac{\sigma_{y_{i}}}{v_{x}} \dot{F}_{y_{i}}+F_{y_{i}}=F_{y_{i_{0}}}
$$

where $i=f, r$ represent respectively the front and rear components. $F_{y_{i}}$ are the lateral tire forces, $\sigma_{y_{i}}$ is the lateral tire relaxation length and $F_{y_{i}}$ are the initial lateral tire forces. To completely define the tire model (1), the terms $F_{y_{i}}$ need to be determined with an adequate expression. It exists many models describing the tire forces, as in [27] where the authors introduced a theoretical approach based on friction theory. Whereas in [8], the author used a semi-empirical method to develop his tire model also well-known under the name of "magic formula". This last is one of the most used because it precisely describes all the tire dynamics phenomena, its parameters can be identified with experimental tests and it can be easily linearized. Finally, for P2WV, the magic formula, in its linear form, is expressed by the following equations:

$$
F_{y_{i}}=C_{i_{1}} \alpha_{i}+C_{i_{2}} \lambda_{i}
$$

where $C_{i_{1}}$ and $C_{i_{2}}$ are respectively the cornering stiffness and camber coefficients of the tire $i$. The side slip angles $\alpha_{i}$ are approximated with the linear expressions:

$$
\left\{\begin{aligned}
\alpha_{f} & =\frac{v_{y}+l_{f} \dot{\psi}-\eta \dot{\delta}}{v_{x}}-\delta \cos (\varepsilon) \\
\alpha_{r} & =\frac{v_{y}-l_{r} \dot{\psi}}{v_{x}}
\end{aligned}\right.
$$

whereas the camber angles $\lambda_{i}$ are linearly given by:

$$
\left\{\begin{array}{l}
\lambda_{f}=\phi+\delta \sin (\varepsilon) \\
\lambda_{r}=\phi
\end{array}\right.
$$

For more details about the derivation of the linear expressions, please refer to [9].

Finally, we get a set of 6 dynamics equations: - Lateral motion:

$$
m_{33} \dot{v}_{y}+m_{34} \ddot{\psi}+m_{35} \ddot{\phi}+m_{36} \ddot{\delta}=r_{34} v_{x} \dot{\psi}+F_{y_{f}}+F_{y_{r}}
$$

- Yaw motion:

$$
\begin{aligned}
m_{34} \dot{v}_{y}+\quad m_{44} \ddot{\psi} \quad+m_{45} \ddot{\phi}+m_{46} \ddot{\delta}=r_{44} v_{x} \dot{\psi}+r_{45} v_{x} \dot{\phi} \\
+r_{46} v_{x} \dot{\delta}+r_{47} F_{y_{f}}+r_{48} F_{y_{r}}
\end{aligned}
$$

- Roll motion:

$$
\begin{aligned}
m_{35} \dot{v}_{y}+m_{45} \ddot{\psi} \quad & +m_{55} \ddot{\phi}+m_{56} \ddot{\delta}=r_{51} \phi+r_{52} \delta+r_{54} v_{x} \dot{\psi} \\
& +r_{56} v_{x} \dot{\delta}
\end{aligned}
$$

- Steering motion:

$$
\begin{aligned}
m_{36} \dot{v}_{y}+\quad m_{46} \ddot{\psi} \quad+m_{56} \ddot{\phi}+m_{66} \ddot{\boldsymbol{\delta}}=r_{61} \phi+r_{62} \delta+r_{64} v_{x} \dot{\psi} \\
+r_{65} v_{x} \dot{\phi}+r_{66} \dot{\delta}+r_{67} F_{y_{f}}+\tau
\end{aligned}
$$


- Front tire motion:

$$
\dot{F}_{y_{f}}=r_{71} v_{x} \phi+r_{72} v_{x} \delta+r_{73} v_{y}+r_{74} \dot{\psi}+r_{76} \dot{\delta}+r_{77} v_{x} F_{y_{f}}
$$

- Rear tire motion:

$$
\dot{F}_{y_{r}}=r_{81} v_{x} \phi+r_{83} v_{y}+r_{84} \dot{\psi}+r_{86} \dot{\delta}+r_{88} v_{x} F_{y_{r}}
$$

Please refer to [28] to get the explicit expressions of the coefficients $m_{i j}$ and $r_{i j}$.

The set of equations (5)-(10) can be transformed into matrix formalism. This leads to the following Linear Parameter Varying (LPV) state-space representation:

$$
\dot{\bar{x}}=\bar{A}\left(v_{x}\right) \bar{x}+\bar{B} \tau
$$

where $\bar{x}=\left[\phi, \delta, v_{y}, \dot{\psi}, \dot{\phi}, \dot{\delta}, F_{y_{f}}, F_{y_{r}}\right]^{T}$ denotes the vector of states, $\bar{A}\left(v_{x}\right)$ the state matrix which depends on the time varying forward speed $v_{x} . \bar{B}$ is the input vector and $\tau$ is the rider's steering torque.

\section{B. Augmented Model}

The rider torque applied on the handlebar denoted $\tau$ is unmesureable. Indeed, even if strain gauges can give an idea about this torque, it is currently impossible to identify the distribution between the pure rider's action and the road aligning moment contribution. In this context, several complex approaches were investigated as in [21] and [25]. In the latter, the authors proposed the use of an unknown input observers to bypass the unmeasurability of $\tau$. Finally, without any information on $\tau$ in the model (11), it is not possible to directly implement a Luenberger observer. In practice, the rider torque is naturally continuous and bounded because it is a human action. It means that its first derivative always exist and is denoted $\dot{\tau}$. Hence, if we augment the state vector $\bar{x}$ with the state $\tau$, then this turns out to be a great solution to bypass the lack of information about $\tau$. In order to express the augmented system in an exact form, the rider torque dynamics is added to the previous system 11 as it follows:

$$
\dot{x}=A\left(v_{x}\right) x+P p
$$

where $x=\left[\phi, \delta, v_{y}, \dot{\psi}, \dot{\phi}, \dot{\delta}, F_{y_{f}}, F_{y_{r}}, \tau\right]^{T}$ denotes the augmented vector of states and $p=\dot{\tau}$ is the rider torque dynamics. The matrices $A\left(v_{x}\right)$ and $P$ are defined as follows:

$$
A\left(v_{x}\right)=\left[\begin{array}{cc}
\bar{A}\left(v_{x}\right) & \bar{B} \\
0_{1 \times 8} & 0
\end{array}\right] \text { and } P=\left[\begin{array}{c}
0_{8 \times 1} \\
1
\end{array}\right]
$$

\section{Exact T-S model of the augmented model}

The well-known sector nonlinearity approach presented in [29] is one of the most used to deal with nonlinear problem. This last allows to describe nonlinear system with a whole of linear sub-systems extending the use of many technical tools originally dedicated to linear problems. It is important to note that this technique keeps an exact expression of the initial nonlinear system in a compact set of the state space set. It does not need any assumption except that the nonlinearities have to be bounded. Nevertheless, for $n$ nonlinearities we get $r=2^{n}$ sub-models. For models with multiple nonlinearities, this rapidly leads to a plenty of expressions. In our case, the Takagi-Sugeno (TS) method allows to take into account the longitudinal speed variation during the observer design.

By following the TS approach, the system (12) can be exactly expressed in a polytopic form as follows:

$$
\dot{x}=\sum_{i=1}^{r=2} \mu_{i}\left(v_{x}\right) A_{i} x+P p
$$

Note that the time varying longitudinal velocity $v_{x}$ is the only nonlinearity $(n=1)$. Hence, the system (12) can be described with 2 linear sub-models. The variables $\mu_{i}($.$) are the weighting$ functions also called the membership functions which are computed as it follows:

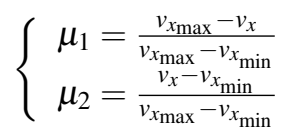

and they must satisfy the following convex sum property:

$$
\left\{\begin{array}{l}
\sum_{i=1}^{r=2} \mu_{i}\left(v_{x}\right)=1 \\
0 \leq \mu_{i}\left(v_{x}\right) \leq 1 ; \quad i=1,2
\end{array}\right.
$$

At this point, the TS approach applied on the augmented system (12) allows us to implement a nonlinear Luenberger observer whose the design is discussed in the next section.

\section{OBSERVER DESIGN}

This section aims to remind the main steps concerning the design of the nonlinear Luenberger observer initially introduced in [20].

\section{A. Observation equation}

Before designing the observer we need to clearly define the measured states. The observer existence conditions are highly correlated to this choice. Regarding the high interest of the motorcycle makers to embed more and more electronics (sensors, ECU, etc), it is consistent and common to consider that the P2WV is equipped with a gyroscope and an accelerometer. Let us consider these sensors are installed as close as possible to the gravity center and provide the measures of the angular pitch, yaw, roll rates, the lateral and vertical accelerations. It is important to note that these measures are given in the bodyfixed frame rigidly attached to the vehicle in contradiction with the reference frame used to derive the model. Hence, the latter are respectively denoted $\dot{\theta}_{b f}, \dot{\psi}_{b f}, \dot{\phi}_{b f}, a_{y_{b f}}$ and $a_{z_{b f}}$.

Like $a_{y}$ is not an explicit state of the model, the following expression $M a_{y}=F_{y f}+F_{y r}$ is introduced with $M=M_{f}+M_{r}$ the total mass. Nevertheless, there is a subtlety because the accelerometer provides the measures in the body-fixed frame whereas $a_{y}$ is the lateral acceleration in the reference frame which is not affected by the roll motion. Consequently, it is necessary to express $a_{y}$ as a function of the body-fixed measures. Under the assumption that the pitch dynamics is neglected as in the development of the Sharp' model, the measured accelerations in the body-fixed frame along $Y$-axis and $Z$-axis can be expressed by:

$$
\left\{\begin{array}{l}
a_{y_{b f}}=a_{y} \cos (\phi)-g \sin (\phi) \\
a_{z_{b f}}=-a_{y} \sin (\phi)-g \cos (\phi)
\end{array}\right.
$$


Although, the lateral accelerations $a_{y_{b f}}$ and $a_{y}$ are expressed in different frames, they systematically have the same sign. If we square the two equations (16) and we sum each other. Then, the lateral acceleration in the reference frame $a_{y}$ can be expressed by the following expression:

$$
a_{y}=\operatorname{sign}\left(a_{y_{b f}}\right) \sqrt{a_{y_{b f}}^{2}+a_{z_{b f}}^{2}-g^{2}}
$$

A similar problem affects the yaw and roll rates respectively denoted $\dot{\psi}$ and $\dot{\phi}$. Indeed, the gyroscope provides the angular rate measurements in the body-fixed frame whereas the state used in the observation equation are in the reference frame. The kinematics of moving frames was largely addressed in the literature as in [?]. The reader can refer to this last for more details on the transformation between the angular rates in the body-fixed and the reference frame. According to [?], the roll, pitch and yaw rates given in the reference frame respectively denoted $\dot{\phi}, \dot{\theta}$ and $\dot{\psi}$ can be expressed by:

$$
\left[\begin{array}{c}
\dot{\phi} \\
\dot{\theta} \\
\dot{\psi}
\end{array}\right]=\left[\begin{array}{ccc}
1 & \sin (\phi) \tan (\theta) & \cos (\phi) \tan (\theta) \\
0 & \cos (\phi) & -\sin (\phi) \\
0 & \sin (\phi) / \cos (\theta) & \cos (\phi) / \cos (\theta)
\end{array}\right]\left[\begin{array}{c}
\dot{\phi}_{b f} \\
\dot{\theta}_{b f} \\
\dot{\psi}_{b f}
\end{array}\right]
$$

Let us consider again the assumption that the pitch dynamics is neglected and the gyroscope is properly calibrated. In other words $\dot{\theta}=0$ and $\theta=0$. Then, using the exactly the same technique as above between (16)-(17) for the two last equations of the system, we obtain:

$$
\dot{\psi}=\operatorname{sign}\left(\dot{\psi}_{b f}\right) \sqrt{\dot{\theta}_{b f}^{2}+\dot{\psi}_{b f}^{2}}
$$

Under the above assumptions, the expression of the roll rate in the reference frame is trivial and given by:

$$
\dot{\phi}=\dot{\phi}_{b f}
$$

Finally, we will consider $a_{y}, \dot{\psi}$ and $\dot{\phi}$ as measured in the observation equation since their algebraic expressions only depend on the gravity acceleration $g$, the accelerometer measurements $\left(a_{y_{b f}}\right.$ and $a_{z_{b f}}$ ) and the gyroscope measurements $\left(\dot{\phi}_{b f}, \dot{\theta}_{b f}\right.$ and $\left.\dot{\psi}_{b f}\right)$.

In addition, the steering angle $\delta$ is also measured. To that end, let us consider that the P $2 \mathrm{WV}$ is equipped with a simple encoder installed on the steering axis. Moreover, efficient numerical differentiation techniques were recently proposed as in [30]. This approach allows to estimate the first and second time derivatives of a given function. In our case, it allows to estimate the steering dynamics $\dot{\delta}$.

Finally, the observation equation is given by:

$$
y=C x+D d
$$

where $y$ is the vector of measures defined by: $y=$ $\left[\delta, \dot{\psi}, \dot{\phi}, \dot{\delta}, a_{y}\right]$ with $a_{y}=\left(F_{y f}+F_{y r}\right) / M$ and $C$ is the corresponding observation matrix. Note that the inertial measurements in $y$ are expressed in the reference frame. Nevertheless, they can be rewritten as a function of the sensor measures as in equations (17)-(20). In order to be as close as possible to a real experimentation, sensor noises are considered. To that end, $d$ denotes the noise vector whereas $D$ is the corresponding matrix.
Finally, these five measures ensure the strong observability of the lateral model whatever the longitudinal speed except for $v_{x}=0$. Nevertheless, in the context of this work we assume that $v_{x}>0$ because the objectives are to develop safety system for forward riding scenario. Backward riding and static maneuvers are not considered here since they are not the most dangerous situations.

\section{B. Observer synthesis}

Let us consider the general TS model:

$$
\left\{\begin{array}{rlc}
\dot{x} & = & \sum_{i=1}^{r} \mu_{i}(\rho) A_{i} x+P p \\
y & = & C x+D d
\end{array}\right.
$$

where $x \in \mathbb{R}^{n}, p \in \mathbb{R}^{n_{p}}, y \in \mathbb{R}^{n_{y}}$ and $d \in \mathbb{R}^{n_{d}}$ are respectively the vector of states, the vector of perturbations, the vector of measures and the vector of disturbances. Note that this last contains the measurement noises. $r$ is the number of submodels given by $r=2^{n}$ with $n$ the number of nonlinearities and $\mu_{i}($.$) are the membership functions. \rho \in \mathbb{R}^{n_{\rho}}$ denotes the premise variable which is considered as measurable for the following section.

Consider the well-known Luenberger observer in its TS form:

$$
\left\{\begin{array}{lcc}
\dot{\hat{x}} & = & \sum_{i=1}^{r} \mu_{i}(\rho)\left(A_{i} \hat{x}+L_{i}(y-\hat{y})\right) \\
\hat{y}= & C \hat{x}
\end{array}\right.
$$

with $L_{i}$ the observer gain matrices which ensure the error convergence. The estimated state and output vectors are respectively denoted $\hat{x}$ and $\hat{y}$. Now, let us consider the state estimation error as follows:

$$
e=x-\hat{x}
$$

Its dynamics is given by the following expressions:

$$
\begin{aligned}
\dot{e} & =\dot{x}-\dot{\hat{x}} \\
& =\sum_{i=1}^{r} \mu_{i}(\rho) A_{i} x+P p-\sum_{i=1}^{r} \mu_{i}(\rho)\left(A_{i} \hat{x}+L_{i}(y-\hat{y})\right) \\
& =\sum_{i=1}^{r} \mu_{i}(\rho)\left(\mathscr{A}_{i} e-L_{i} D d\right)+P p \\
& =\sum_{i=1}^{r} \mu_{i}(\rho)\left(\mathscr{A}_{i} e-S_{i} s\right)
\end{aligned}
$$

with $\mathscr{A}_{i}=A_{i}-L_{i} C, S_{i}=\left[\begin{array}{ll}P & -L_{i} D\end{array}\right]$ and $s=\left[\begin{array}{ll}p^{T} & d^{T}\end{array}\right]^{T}$

Then, in order to analyze the error convergence, let us introduce the following Lyapunov function denoted $V$ :

$$
V=e^{T} X e
$$

with $X$ a symmetric and positive definite matrix such that: $X=X^{T}>0$.

Its time derivative $\dot{V}$ leads to:

$$
\dot{V}=\sum_{i=1}^{r} \mu_{i}(\rho)\left(e^{T}\left(\mathscr{A}_{i}^{T} X+X \mathscr{A}_{i}\right) e+s^{T} S_{i}^{T} X e+e^{T} X S_{i} s\right)
$$

To attenuate the effect of the perturbation $s$ on the estimation error $e$, let us define the $L_{2}$-gain as the quantity:

$$
\sup _{\|s\|_{2} \neq 0} \frac{\|e\|_{2}}{\|s\|_{2}} \leq \gamma^{2}
$$


with $\gamma$ a positive scalar and $\|.\|_{2}$ the $L_{2}$-norm. For a given vector $z(t)$, its $L_{2}$-norm is:

$$
\|z(t)\|_{2}=\left(\int_{0}^{\infty} z^{T}(t) z(t) d t\right)^{1 / 2}
$$

Hence, it comes the following inequality:

$$
e^{T} e-\gamma^{2} s^{T} s<0
$$

Considering that $\dot{V}<0$, it follows:

$$
\dot{V}+e^{T} e-\gamma^{2} s^{T} s<0
$$

Note that even with the presence of a disturbance term $s$, if the inequality (31) is verified then the convergence of the estimation error is ensured.

Considering the notation $\tilde{Z}_{i}=\sum_{i=1}^{r} \mu_{i}(\rho) Z_{i}$, the expression of $\dot{V}$ given in (27) and the equation (31), we obtain a Linear Matrix Inequality (LMI) problem:

$$
\left[\begin{array}{c}
e \\
s
\end{array}\right]^{T}\left[\begin{array}{cc}
\tilde{A}_{i}^{T} X+X \tilde{\mathscr{A}}_{i}+I & X \tilde{S}_{i} \\
\tilde{S}_{i}^{T} X & -\gamma^{2} I
\end{array}\right]\left[\begin{array}{l}
e \\
s
\end{array}\right]<0
$$

Since the weighting functions satisfy the convex sum property, sufficient conditions ensuring the convergence of estimation error are given by:

$$
\left[\begin{array}{cc}
\mathscr{A}_{i}^{T} X+X \mathscr{A}_{i}+I & X S_{i} \\
S_{i}^{T} X & -\gamma^{2} I
\end{array}\right]<0, \quad i=1, \ldots, r
$$

In order to obtain solvable LMI conditions, the changes of variable $\bar{L}_{i}=X L_{i}, A_{i}=\mathscr{A}_{i}+L_{i} C$ and $\bar{\gamma}=\gamma^{2}$ are necessary. It leads to the final LMI:

$$
\left[\begin{array}{cc}
A_{i}^{T} X+X A_{i}-\bar{L}_{i} C-C^{T} \bar{L}_{i}^{T}+I & X S_{i} \\
S_{i}^{T} X & -\bar{\gamma} I
\end{array}\right]<0, \quad i=1, \ldots, r
$$

Finally, given a scalar $\gamma$, if there exists a symmetric and positive definite matrix $\mathrm{X}$ and matrices $\bar{L}_{i}, i=1, \ldots, r$ such that the LMI (34) is satisfied. Then the error is stable and the transfer from the perturbation $s$ to the estimation error $e$ is bounded by $\gamma$. Note that the observer gain matrix $L$ is obtained by:

$$
L=\sum_{i=1}^{r} \mu_{i}(\rho) X^{-1} \bar{L}_{i}
$$

In practice, to get better estimation performance, it is possible to transform the previous LMI (34) into an optimization problem. To that end, $\gamma$ can be considered as an adjustable parameter of optimization that the designer can control.

\section{OBSERVER VALIDATION}

This section deals with the main contribution of this paper. Indeed, this observer was already introduced in [20] but the algorithm was only tested on the two-body linear model used for the observer design. This two-body model is quite simple and based on many assumptions (linear dynamics, rigid bodies, pitch dynamics neglected, etc.). Although, the results discussed in [20] highlight very good estimation performances. This does not mean that it will be as efficient in a realistic case because of the restrictive assumptions. Moreover, our previous work considered ideal sensors. It did not take into account any measurment noise during the observer design. To go further, this paper presents a more realistic validation since it proposes to test the observer by means of the commercial motorcycle simulator BikeSim while considering realistic sensors. This software is commonly used in P2WV research and development field as in [31], [32] or in [25]. It is a complete simulator which is based on the multibody model introduced in [5]. where the authors modelled the motorcycle as a set of 10 different bodies. This model allows a total of 29 DOF leading to a very complex and highly nonlinear equations. Even if no real experiment is carried out, BS allows us to test the observer in conditions very close to a real riding test. In addition, simulators like BS turn out to be more and more attractive since they allow to test dangerous situations without any real risk for the rider. To conclude the validation, the observer performances are quantified with the RMSE criteria and compared to similar works.

Let us remind that the objective of this observer is to estimate the non-measured motorcycle lateral states and the rider action applied on the handlebar whatever the longitudinal speed. To that end, the estimated states are the roll angle $\phi$, the lateral speed $v_{y}$, the lateral tire forces $F_{y_{f}}, F_{y_{r}}$ and the rider torque $\tau$. Note that the lateral speed estimation is not addressed below. The first reason is that the lateral speed is a better indicator for comfort than for critical situation detection like a rollover scenario. In this last case, other states like the roll angle and its rate are much more important. The second is that $v_{y}$ is lowly excited leading to significant estimation errors.

The observer is tested on three distinct scenarios. A first one which represents a track at varying forward speed in order to simulate a daily life riding. Then, a second and a third scenarios whose the aims are to test the observer on extreme maneuvers which highly excite the lateral motorcycle dynamics. They simulate respectively a slalom with a simultaneous acceleration and a Double Lane Change (DLC) at constant speed with noisy sensors.

As discussed in the observer design section, we turner the LMI (34) into an optimization problem by assuming $\gamma$ as a chosen optimization parameter. Finally, we set $\gamma=0.0464$ which ensures a excellent compromise between estimation performances and computing resources. Indeed, with this value of $\gamma$, the algorithm is largely able to perform real time estimations without any hardware optimization at that time. Note that, the tests was carried out on macOS with a $3.1 \mathrm{GHz}$ Intel Core i7 CPU.

\section{A. Track scenario}

With its straight lines, its large and narrow turns and its rapid speed variations included between 30 and $100 \mathrm{~km} / \mathrm{h}$, 


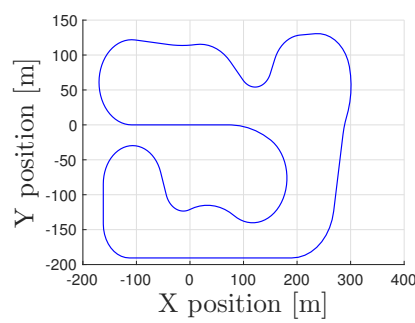

(a) Vehicle trajectory

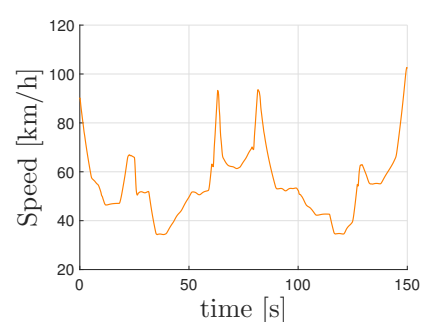

(b) Forward speed
Figure 2: Track scenario

the riding track scenario introduced in figure 2 is very representative to common riding situations. The objective of this first simulation is to test the ability of the proposed observer along a scenario as close as possible to a daily life use of a P2WV.

As discussed above, accelerometers, gyroscopes and encoders became affordable and common sensors in vehicle embedded instrumentation. In our case, these three sensors guarantee the observability conditions of the system (12). Let us recall that the measures states $\dot{\psi}, \dot{\phi}$ and $a_{y}$, in the observation equation (21) are expressed in the reference frame whereas the sensors provide the measures in the body-fixed frame. They are considered as measured since their algebraic expressions only depends on the measurements as explained in the equations (17)-(20). Note that the encoder gives directly the measure of the steering angle $\delta$ without any frame transformation. In addition, a prior differentiator allows to estimate its dynamics $\dot{\delta}$ like in [28].

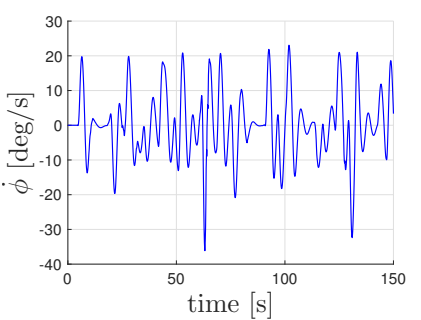

(a) Measured roll rate

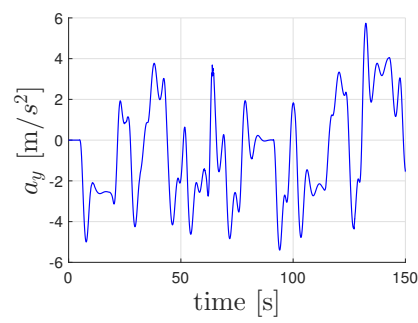

(c) Measured lateral acceleration

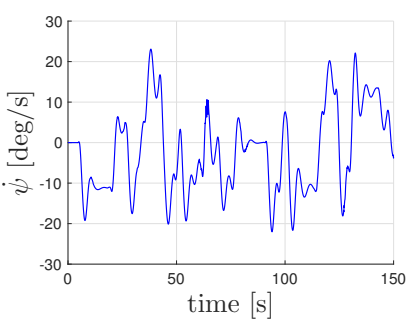

(b) Measured yaw rate

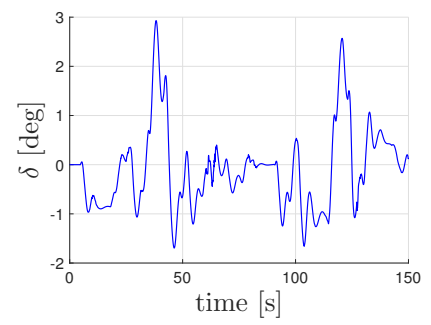

(d) Measured steering angle

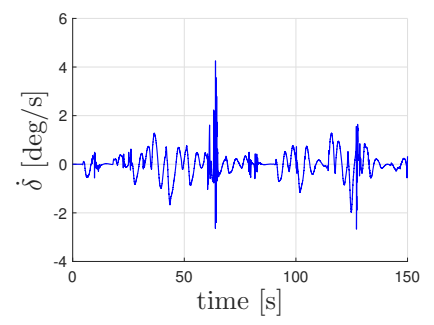

(e) Measured steering rate

Figure 3: Measured states along the track scenario

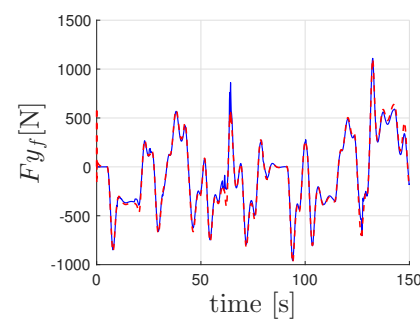

(a) Actual (blue) and estimated (red) front tire force

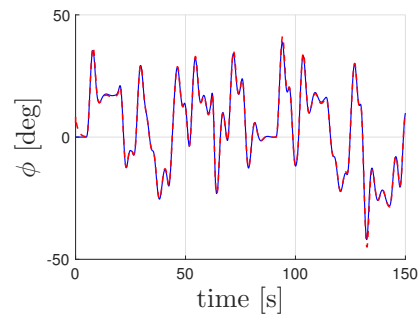

(c) Actual (blue) and estimated (red) roll angle

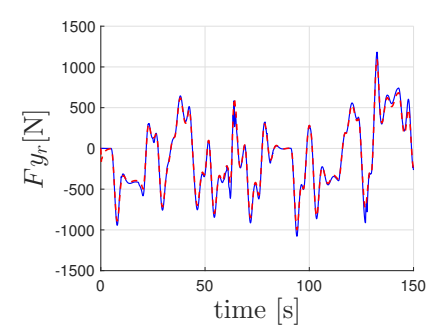

(b) Actual (blue) and estimated (red) rear tire force

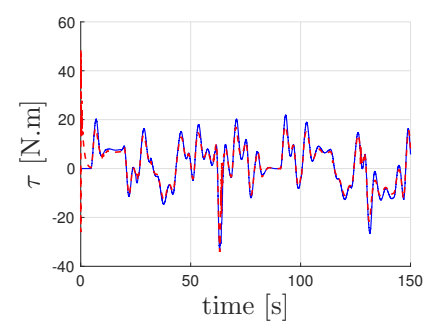

(d) Actual (blue) and estimated (red) rider's torque
Figure 4: Estimated states along the track scenario

Figure 3 introduces the measured states along the track. One can remark the small value of the steering regarding some turns. this endorses that the lateral control of a P2WV is the result of several complex actions (handlebar steering, vehicle leaning, etc.). 
Figure 4 presents the lateral dynamics states and the rider torque simulated in blue and their estimation in red. It clearly shows all the capabilities of the nonlinear Luenberger observer to estimate the rider action and the motorcycle lateral dynamics. Note that, the observer initial conditions were willingly chosen different from the model to show the ability to rapidly converge toward the simulated value.

\section{B. Extreme slalom test}

In contrast to the first scenario, the slalom aims to simulate an extreme riding maneuver since it highly excites the lateral dynamics. Moreover, the forward speed increases simultaneously with the lateral rider action in order to test the estimation performances independently of the longitudinal speed variations. Figure 5 depicts the lateral trajectory and the simultaneous acceleration from 50 to $100 \mathrm{~km} / \mathrm{h}$.

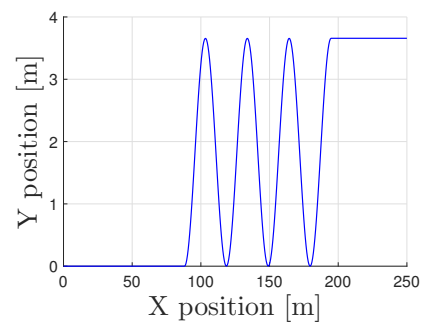

(a) Vehicle trajectory

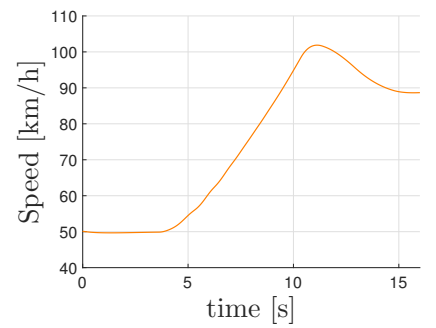

(b) Forward speed

Figure 5: Slalom scenario

Figure 6 introduces the measured states during the slalom test. Whereas in figure 7 , the simulated dynamics states are plotted in blue and their estimation in red. One can remark that the successive rider torque peaks which illustrate the high dynamics solicitations. As for the track case, the initial conditions were chosen different from the initial dynamics states of the P2WV. This scenario endorses that this observer is able to estimate the lateral dynamics and the rider action even for extreme riding scenarios. Nevertheless, some small estimation errors are visible especially in the peak areas. Let us remind that the observer is derived from a linear twobody model whereas BS is a highly nonlinear multi-body simulator which could explain these errors. In addition, in the observer algorithm the lateral tire forces are approximated by their linear expressions leading to significant errors when the saturation phenomenon appears.

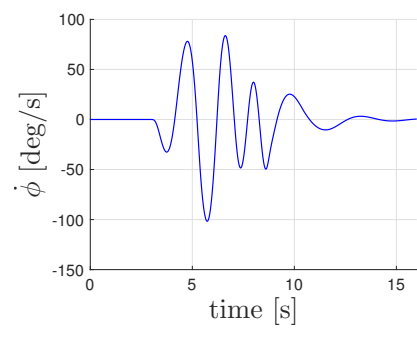

(a) Measured roll rate

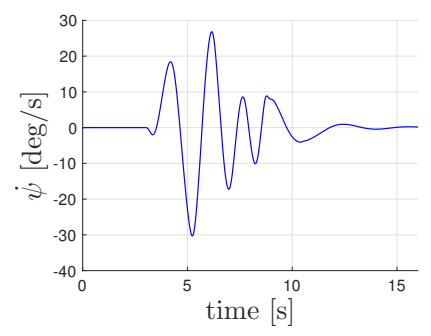

(b) Measured yaw rate

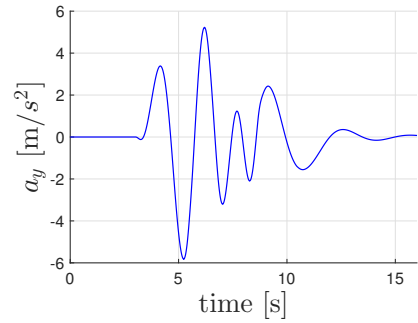

(c) Measured lateral acceleration

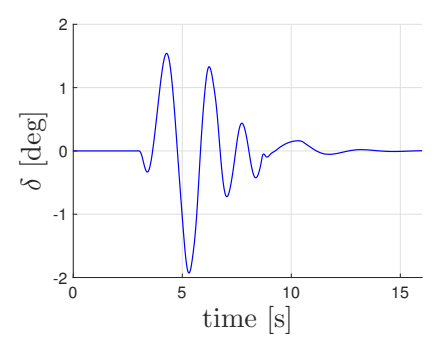

(d) Measured steering angle

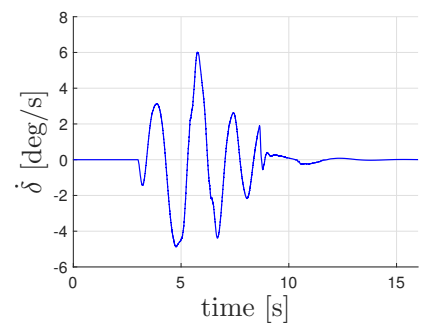

(e) Measured steering rate

Figure 6: Measured states during the slalom

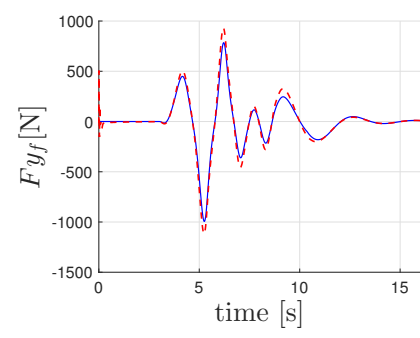

(a) Actual (blue) and estimated (red) front tire force

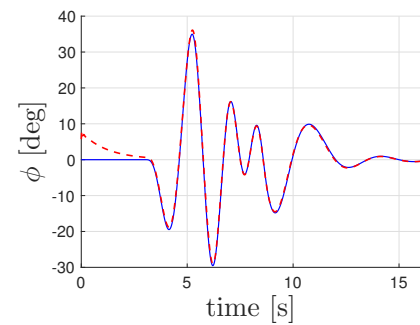

(c) Actual (blue) and estimated (red) roll angle

Figure 7: Estimated states during the slalom

\section{Double lane change test with noisy sensors}

This section aims to test the robustness of the observer regarding sensor noises. Moreover, a double lane change (DLC) scenario at high speed is introduced. Note that the DLC which, in practice, represents an avoidance maneuver is among the most important riding cases to test the algorithm performances for safety system applications. Hence, behind this section there are two objectives, the first is to test the sensor noise sensibility and the second to demonstrate the observer capabilities in a typical dangerous riding situation. Figure 8 shows the motorcycle trajectory during the DLC whereas the forward speed $v_{x}$ is constant at $100 \mathrm{~km} / \mathrm{h}$. 


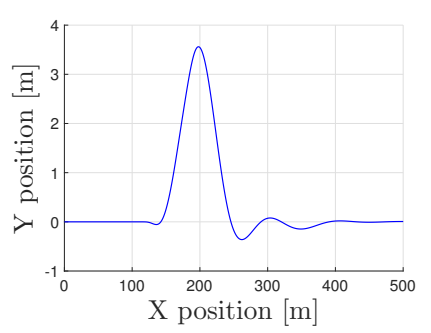

Figure 8: DLC scenario

Although is able to faithfully simulate the whole of motorcycle dynamics, the sensor imperfections are not taken into account. To be as close as possible to an experimental validation a centered white noise has been added on each measure such that the noise is bounded between 5 and 10 $\%$ of the maximum value of the considered measure.

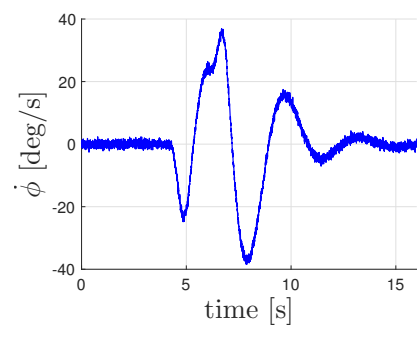

(a) Measured roll rate with noise

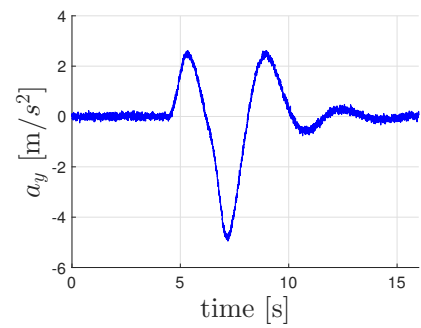

(c) Measured lateral acceleration with noise

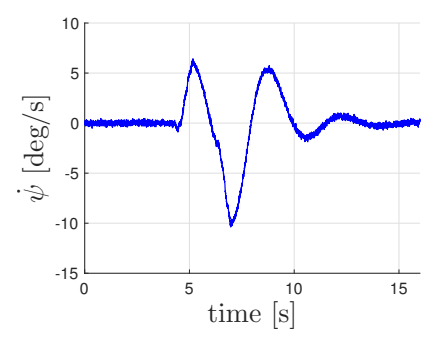

(b) Measured yaw rate with noise

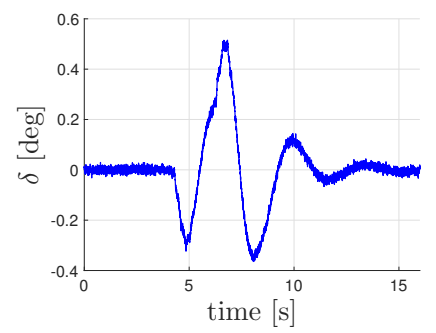

(d) Measured steering angle with noise

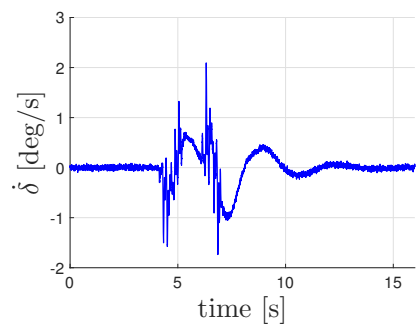

(e) Measured steering rate with noise

Figure 9: Measured states with noise during the DLC

Figure 10 presents the measures perturbed with the sensor noise. In contrast with the two previous simulations were the steering angle was up to 2 degrees, for the DLC in figure 8 it is included between -0.5 and 0.5 degree although the lateral displacement is significant. This observation highlights the correlation between the rider action on the handlebar and the forward speed. In practice, to negotiate a turn a rider has to increase the steering angle as far as the forward speed is slowing down.

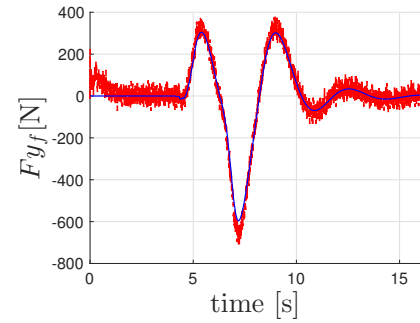

(a) Actual (blue) and estimated (red) front tire force

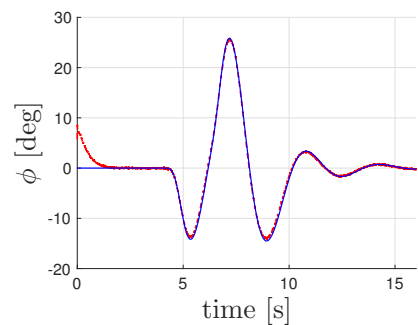

(c) Actual (blue) and estimated (red) roll angle

Figure 10: Estimated states during the DLC

Figure 10 shows the consequences of noises on the estimations of the lateral dynamics and rider action. One can clearly remark that the rider torque is the most affected estimated state and consequently the front lateral tire force. The rear tire force and the roll angle are practically insensitive to sensor noises, the observer acts as a real filter for these two estimated states. The different noise sensitivities between front and rear tire forces are explained by the fact that the steering dynamics mostly affects the front tire dynamics as it is expressed in (9).

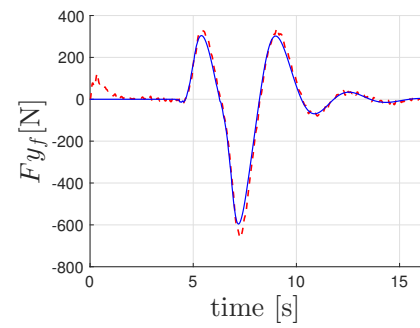

(a) Actual (blue) and filtered estimated (red) front tire force

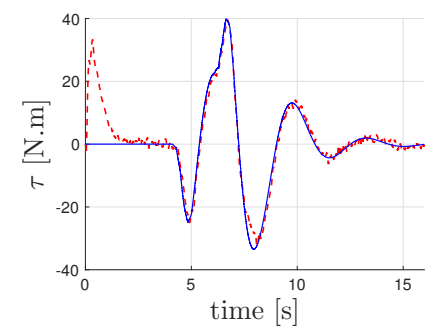

(b) Actual (blue) and filtered estimated (red) rider's torque
Figure 11: Estimated states during the DLC

Nevertheless, signal processing techniques such as a lowpass filter turns out to be an efficient solution in order to attenuate the effect of the noises on the estimations. A third order Butterworth filter with adequate cut-off frequency is sufficient to denoise the data without leading to a significant delay. Figure 11 presents the filtered estimated rider torque and the obtained front tire force.

\section{Observer performances quantification}

In [26] or [25], authors have already proposed a solution to quantify the performances of their observer through the root mean square error comparison. Nevertheless, these RMSE 
studies was only devoted to roll angle estimation. Let us remind that the RMSE is defined by:

$$
R M S E=\sqrt{\frac{1}{n} \sum_{i=1}^{n}\left(y_{i}-\hat{y}_{i}\right)^{2}}
$$

Considering that the RMSE is proportional to the square of the estimation error, less is the RMSE better are the estimation performances.

In [26] authors have proposed to compare roll angle estimation performances thanks to a vision system, a Kalman filter based on an inertial measurement unit (IMU) and the combination of the two methods. The approaches are tested on an experimental track scenario. Whereas in [25], the performances of an Unknown Input Observer (UIO) have been presented for two cases, a first with ideal sensors and a second with sensor noise consideration. In this work, the author tested his observer through track scenario simulations with . Note that the scenario used to compute the RMSE in [25] is exactly the same as in section V.A. The results of these two papers are summarized below in table II.

\begin{tabular}{|c|c|}
\hline Method & RMSE \\
\hline Vision system, [26] & 2.24 \\
\hline IMU-Kalman filter, [26] & 2.01 \\
\hline Mean vision/IMU, [26] & 1.20 \\
\hline UIO with ideal sensors, [25] & 1.28 \\
\hline UIO with realistic sensors, [25] & 1.85 \\
\hline
\end{tabular}

Table II: RMSE results from previous works

Finally, table III introduces the performance of the Luenberger nonlinear observer. For each scenario, the RMSE of the roll angle estimation is computed in a first time with ideal sensor free of noise and then by taking into account sensor noises. For the noisy case the RMSE is computed from the raw roll angle estimation, it means that no prior filter has been used to denoise the data.

The RMSE results for the track scenario demonstrates the potential of our observer compared to previous works. Indeed, in [25], the authors have already tested their UIO on the same track scenario with ideal and realistic sensors. Without any noise consideration the performances of the Luenberger observer are $38 \%$ better and with noisy measures around $54 \%$. Although in their paper the authors have used a more complex alternative to the two-body linear model by taking into account the nonlinearity of the roll and steering angle to be closer to the real dynamics, the performances of our observer, only based on the simple linear model, are better.

Regarding table III, the poorer RMSE results obtained for the slalom and the DLC scenarios highlight the greater difficulty to perfectly estimate the roll angle during extreme maneuvers than for a normal riding case like the track simulation. The modeling assumptions are responsible to estimation error especially the linear approximation of the motorcycle and the tire models. Note that even if the RMSE results of these two scenarios are higher, they are better than those obtained in table II.

Finally, the small variations of the RMSE results between noise free and realistic sensors cases for a same scenario illustrates the prior observation about the fact that the roll angle estimation is almost insensitive to the sensor noise.

\begin{tabular}{|c|c|c|}
\hline & \multicolumn{2}{|c|}{ RMSE } \\
\hline Scenario & Ideal sensors & Real sensors \\
\hline Track & 0.79 & 0.84 \\
\hline Slalom & 1.09 & 1.53 \\
\hline DLC & 1.08 & 1.55 \\
\hline
\end{tabular}

Table III: RMSE results for the proposed Luenberger observer

\section{CONCLUSION}

This paper has presented a concrete validation of the nonlinear Luenberger observer through simulations performed with the commercial simulator BikeSim. Moreover, realistic sensors have been considered during the design to synthesis a robust observer against measurment noises. The lateral vehicle dynamics basis and the main step of the observer design have been reminded. The Lyapunov theory, the LMI tools and the L-2 gain property applied on the Sharp' 71 linear two-body model have contributed to fully design the robust observer and ensure a bounded estimation error with the ISS property. The approach has shown that even if the observer design is based on a simple linear two-body model and the convergence is only bounded, it is able to take into account all the complexity of the motorcycle motion and to perfectly estimate in the same time the lateral dynamics states of the P2WV and the rider action. Then, a slalom and a DLC maneuvers have demonstrated the observer robustness regarding extreme lateral riding scenarios and realistic sensor consideration. Finally a RMSE study, has shown the impressive performances of the proposed observer in comparison with the two previous works [26] and [25].

The future work will consist in real time validation of this observer through experimental tests.

\section{REFERENCES}

[1] M. M. A. Manan and A. Varhelyi, "Motorcycle fatalities in malaysia," IATSS Research, vol. 36, no. 1, pp. 30 - 39, 2012.

[2] WHO, "Global status report on road safety 2015," World Health Organization, Tech. Rep., 2015.

[3] ONISR, "La sécurité routière en france: Bilan de l'accidentalité de l'année 2015," ONISR (Observatoire National Interministeriel de la Sécurité Routière), Tech. Rep., 2015.

[4] ACEM, "Motorcycle accidents in depth study (maids)," ACEM (European Association of Motorcycle Manufacturers), Tech. Rep., 2009.

[5] R. S. Sharp, S. Evangelou, and D. J. N. Limebeer, "Advances in the modelling of motorcycle dynamics," Multibody system dynamics, vol. 12, no. 3, pp. 251-283, 2004.

[6] L. Nehaoua, H. Arioui, N. Seguy, and S. Mammar, "Dynamic modeling of a two wheeled vehicle: Jourdain formalism," Vehicle System Dynamics, vol. 51, no. 5, pp. 648-670, 2013.

[7] M. Fouka, P. M. Damon, L. Nehaoua, H. Arioui, and S. Mammar, "Parametric identification of a powered two-wheeled vehicles: Algebraic approach," Mediterranean Conference on Control and Automation (MED), pp. 1047-1052, July 2017.

[8] H. Pacejka, Tire and Vehicle Dynamics. SAE International, 2005.

[9] V. Cossalter, Motorcycle dynamics. Lulu, 2006.

[10] R. S. Sharp, "The stability and control of motorcycles," Journal of Mechanical Engineering Science, vol. 13, no. 5, pp. 316-329, 1971.

[11] U. Nenner, R. Linker, and P.-O. Gutman, "Robust feedback stabilization of an unmanned motorcycle," Control Engineering Practice, 2010.

[12] J. Yi, D. Song, A. Levandowski, and S. Jayasuriya, "Trajectory tracking and balance stabilization control of autonomous motorcycles," in Robotics and Automation, 2006. ICRA 2006. Proceedings 2006 IEEE International Conference on. IEEE, 2006. 
[13] J. Yi, Y. Zhang, and D. Song, "Autonomous motorcycles for agile maneuvers, part i: Dynamic modeling," in Decision and Control, 2009 held jointly with the 2009 28th Chinese Control Conference. CDC/CCC 2009. Proceedings of the 48th IEEE Conference on, 2009.

[14] I. Boniolo, M. Norgia, M. Tanelli, C. Svelto, and S. M. Savaresi, "Performance analysis of an optical distance sensor for roll angle estimation in sport motorcycles," IFAC Proceedings Volumes, 2008.

[15] A. Teerhuis and S. Jansen, "Motorcycle state estimation for lateral dynamics," Vehicle System Dynamics, 2012.

[16] I. Boniolo, S. M. Savaresi, and M. Tanelli, "Roll angle estimation in two-wheeled vehicles," IET Control Theory Applications, vol. 3, no. 1, pp. 20-32, January 2009.

[17] R. Lot, V. Cossalter, and M. Massaro, "Real-time roll angle estimation for two-wheeled vehicles," vol. 1, no. 1, July 2012, pp. 687-693.

[18] D. Ichalal, H. Dabladji, H. Arioui, S. Mammar, and L. Nehaoua "Observer design for motorcycle lean and steering dynamics estimation: A Takagi-Sugeno approach," in American Control Conference (ACC), June 2013, pp. 5654-5659.

[19] M. E.-H. Dabladji, D. Ichalal, H. Arioui, S. Mammar, and L. Fridman, "Estimation of lateral dynamics and road curvature for two-wheeled vehicles: A hosm observer approach," vol. 19, no. 1, pp. 2806-2811, 2014.

[20] P. Damon, H. Dabladji, D. Ichalal, L. Nehaoua, and H. Arioui, "Estimation of lateral motorcycle dynamics and rider action with luenberger observer," Intelligent Transportation Systems (ITSC), 2016.

[21] P. M. Damon, H. Dabladji, D. Ichalal, L. Nehaoua, H. Arioui, and S. Mammar, "Lateral motorcycle dynamics and rider action estimation: An lpv unknown input observer approach," in 2016 IEEE Conference on Control Applications (CCA), Sept 2016, pp. 711-716.

[22] H. Dabladji, D. Ichalal, H. Arioui, and S. Mammar, "Unknown-input observer design for motorcycle lateral dynamics: Ts approach," Control Engineering Practice, vol. 54, pp. 12-26, 2016.

[23] P. D. Filippi, M. Tanelli, M. Corno, S. M. Savaresi, and L. Fabbri, "Design of steering angle observers for the active control of twowheeled vehicles," in 2010 IEEE International Conference on Control Applications, Sept 2010, pp. 155-160.

[24] I. Boniolo, S. M. Savaresi, and M. Tanelli, "Lean angle estimation in two-wheeled vehicles with a reduced sensor configuration," in 2012 IEEE International Symposium on Circuits and Systems, May 2012, pp. 2573-2576.

[25] M. E.-H. Dabladji, D. Ichalal, H. Arioui, and S. Mammar, "Unknowninput observer design for motorcycle lateral dynamics: Ts approach," Control Engineering Practice, vol. 54, pp. 12-26, 2016.

[26] M. Schlipsing, J. Salmen, B. Lattke, K. G. Schrter, and H. Winner, "Roll angle estimation for motorcycles: Comparing video and inertial sensor approaches," pp. 500-505, June 2012.

[27] E. Velenis, P. Tsiotras, and C. Canudas-de Wit, "Extension of the lugre dynamic tire friction model to $2 \mathrm{~d}$ motion," in Proceedings of the 10th IEEE Mediterranean Conference on Control and Automation-MED, 2002.

[28] P. M. Damon, D. Ichalal, H. Arioui, and S. Mammar, "Cascaded flatnessbased observation approach for lateral motorcycle dynamics estimation," 2017 IEEE International Conference on Systems, Man, and Cybernetics (SMC), 2017.

[29] K. Tanaka and H. Wang, Fuzzy Control Systems Design and Analysis: A Linear Matrix Inequality Approach. John Wiley and Sons, 2001.

[30] M. Fliess, C. Join, and H. Sira-Ramirez, "Non-linear estimation is easy," International Journal of Modelling, Identification and Control, vol. 4, no. 1 , pp. 12-27, Jan. 2008.

[31] M. Tanelli, C. Vecchio, M. Corno, A. Ferrara, and S. M. Savaresi, "Traction control for ride-by-wire sport motorcycles: A second-order sliding mode approach," IEEE Transactions on Industrial Electronics, 2009.

[32] C. F. H. Y. J. Shiao, Q. A. Nguyen, "Actuation of adaptive rearview system for motorcycles," Applied Mechanics and Materials, 2013.

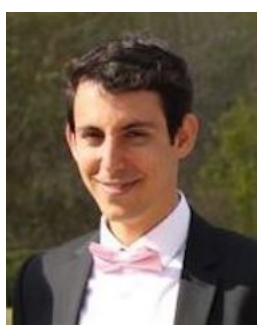

Pierre-Marie Damon received the Dipl.-Ing. and Master's degrees in automotive engineering respectively from ESTACA (Ecole Supérieure des Techniques Aéronautiques et de Construction Automobile) and ENS (Ecole Normale Supérieure) Cachan, Paris, France, in 2014. Since 2015, he works as a $\mathrm{Ph} . \mathrm{D}$ student at IBISC (Informatique, Biologie Intégrative et systmes complexes) Laboratory, Université dEvry Val dEssonne, Evry, France. His research interests deal with motorcycle dynamics, observation and control.

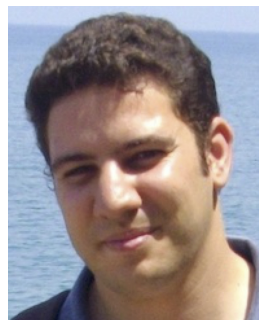

Dalil Ichalal received his Master's degree from Paul Cézanne Aix Marseille 3 University (France) in 2006, and a Ph.D. degree from the National Polytechnic Institute of Lorraine (France) in 2009. In 2010, he joined the Department of Electrical Engineering of the University of Evry Val d'Essonne (France) and the IBISC (Informatique, Biologie Intgrative et systèmes complexes) Laboratory. His research interests include state estimation and observer design, as well as fault diagnosis and fault tolerant control of nonlinear systems with application to vehicles and motorcycles.

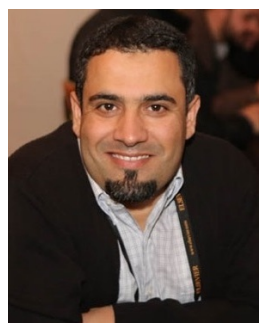

Hichem Arioui received the Dipl.Ing. degree in control systems from the Universite Badji MokhtarAnnaba, Annaba, Algeria, in 1998 and the M.S. and Ph.D. degrees in robotics and automation from the Université dEvry Val-dEssonne (UEVE), Evry, France, in 1999 and 2002, respectively. He was with the Informatics, Integrative Biology and Complex Systems Laboratory, UEVE, where he worked on the control of haptic display under varying time delay in the frame of his Ph.D. dissertation. Since 2003, he has been an Assistant Professor of electrical engineering with the UEVE and since 2010 Associate Professor. His research interest includes modeling and control of haptic interaction, design and control of driving simulator applications and, more recently development of Advanced Rider Assistance System for Motorcycles. 